\title{
Logarithmic Operators in the Theory of Plateau Transition
}

\author{
Ian I. Kogan and Alexei M. Tsvelik \\ Department of Physics, University of Oxford, 1 Keble Road, Oxford, OX1 3NP, UK
}

(July 24, 2021)

We show that $S L(2 ; C) / S U(2)$ model which had been recently proposed to describe the behaviour of the local densities of states at the plateau transition in Integer Quantum Hall effect, has logarithmic operators. They unusual properties are studied in this letter.

OUTP-99-08S PACS numbers: 75.10 Jm, 75.40 Gb.

In our previous paper [1] we have discussed a problem of plateau transition in Integer Quantum Hall effect. We have provided evidence to suggest that the correlation functions of the local densities of states (DOS) at the critical point may be obtained from the critical $S L(2 ; C) / S U(2)$ Wess-Zumino-Novikov-Witten (WZNW) model, in which the level $k^{\prime}=-(k+2)(k>0)$ is fixed by the known scaling dimensions. The action of this model is given by

$$
S_{0}=\frac{(k+2)}{4 \pi} \int d^{2} x\left[4 \partial \theta \bar{\partial} \theta+e^{2 \theta} \partial \mu \bar{\partial} \mu^{*}\right]
$$

This model has numerous applications in field theory including the theory of disorder (see, for example [2] and references therein). Among unconventional features displayed by this model is the presence of non-trivial primary fields with zero scaling dimension. These operators are likely to play very important role in the applications. Thus, in [1] we have suggested that to study correlation functions of local DOS one has to consider the effective action

$$
S=S_{0}+\eta \int d^{2} x \Psi_{0}(\mathbf{x})
$$

where $\Psi_{0}$ is a non-trivial primary field of scaling dimension zero. We have argued that in order to obtain the consistent results for the correlation functions at the critical point, one has to execute the $\eta \rightarrow 0$ limit. This includes expansion in powers of $\eta$ keeping only the first non-vanishing term. In this procedure the two-point correlation function of the $q^{t h}$ powers of the local DOS is expressed as the limit of the four-point correlation function:

$$
\begin{array}{r}
\overline{\rho^{q}\left(\mathbf{r}_{1}\right) \rho^{q}\left(\mathbf{r}_{2}\right)}= \\
\lim _{\left|\mathbf{r}_{3}\right| \rightarrow 0,\left|\mathbf{r}_{4}\right| \rightarrow \infty}\left\langle\rho^{q}\left(\mathbf{r}_{1}\right) \rho^{q}\left(\mathbf{r}_{2}\right) \Psi_{0}\left(\mathbf{r}_{3}\right) \Psi_{0}\left(\mathbf{r}_{4}\right)\right\rangle
\end{array}
$$

where we use the overbar to denote disorder averaging, and use the angular brakets to denote correlation functions of our field theory. Suggesting this procedure we have assumed that $\Psi_{0}$ was a relevant operator such that at any finite $\eta$ its presence in the effective action generates the scale $L_{\eta} \sim \eta^{-1 / 2}$.

In this paper we show that the theory possesses another field with zero scaling dimension which presence in the effective action does not violate the conformal invariance. A similar example has already been considered in [1] (see Appendix 6) where it was shown that at $k=2$ kinetic the energy perturbation model does not violate conformal invariance of model (11)0). The corresponding operator has the Operator Product Expansion (OPE) $\Psi_{0}(z) \Psi_{0}(0)=\Psi_{0}(0)$, and hence has zero norm. Such OPE is essential for preservation of the conformal invariance of the theory.

There is a degeneracy in the present problem: besides the nontrivial operator with dimension zero there is always a unity operator. One can think that the pair of degenerate operators form the logarithmic pair 3. In such a pair one of the operators always has zero norm [4]:

$$
\begin{aligned}
\langle C(x) D(y)\rangle & =\langle C(y) D(x)\rangle=\frac{c}{(x-y)^{2 \Delta_{C}}} \\
\langle D(x) D(y)\rangle & =\frac{1}{(x-y)^{2 \Delta_{C}}}(-2 c \ln (x-y)+d) \\
\langle C(x) C(y)\rangle & =0
\end{aligned}
$$

where in our case $\Delta=0$

The purpose of this letter is to investigate the properties of zero-dimensionmal operators $S L(2, R)$ model and to see if there are logarithmic operators in the theory. Let us note that logarithmic operators in $S L(2, R)$ model have been discussed before (see for example [5]) but not particularly zero dimensional ones.

Primary fields of model (11) are tensors from the representations of the $S L(2, R)$ group. Each representation is labeled by its angular momentum $j$. The conformal weights are

$$
h_{j}=-j(j+1) / k
$$

The multi-point correlation function of primary fields belonging to representations with angular momentum $j_{a}$ is given by the following Knizhnik-Zamolodchikov (KZ) equation 6], 8]:

$$
\begin{gathered}
\left\{k \frac{\partial}{\partial z_{i}}-\sum_{j \neq i}^{N} \frac{L_{i j}}{z_{i j}}\right\} G(1 \cdots N)=0 \\
L_{i j}=\left(y_{i j}\right)^{2} \frac{\partial^{2}}{\partial y_{i} \partial y_{j}}+2 y_{i j}\left(j_{j} \frac{\partial}{\partial y_{i}}-j_{i} \frac{\partial}{\partial y_{j}}\right)-2 j_{i} j_{j}
\end{gathered}
$$


Here the parameter $y$ parametrizes the $S L(2, R)$ group variables and one gets the following representation for the $S L(2, R)$ generators

$$
t^{+}=\frac{\partial}{\partial y}, \quad t^{3}=y \frac{\partial}{\partial y}-j, \quad t^{+}=y^{2} \frac{\partial}{\partial y}-2 j y
$$

In this parametrization a matrix operator $\Phi_{k, \bar{k}}$ is mapped on a function of complex variable $\Phi(y, \bar{y})$ (we omit here the dependence on spatial coordiantes $z$ and $\bar{z}$ ) as

$$
\Phi^{(j),(\bar{j})}(y, \bar{y})=\sum_{k, \bar{k}} y^{j+k} \bar{y}^{\bar{j}+\bar{k}} \Phi_{k, \bar{k}}^{(j),(\bar{j})}
$$

Let us discuss now the case when all operators have $j=0$. The equation (6) for $j=0$ becomes

$$
\left\{k \frac{\partial}{\partial z_{i}}-\sum_{j \neq i}^{N} \frac{1}{z_{i j}}\left[\left(y_{i j}\right)^{2} \frac{\partial^{2}}{\partial y_{i} \partial y_{j}}\right]\right\} G(1 \cdots N)=0
$$

We shall concentrate on holomorphic dependence on $z$ and $y$ only, the restoration of $\bar{z}$ and $\bar{y}$ dependence is straightforward. Let us study first of all the case of $N=2$. The two-point functions $G_{2}(z, y)=<$ $\Psi_{0}(z, y) \Psi_{0}(0,0)>$ which obeys the equation

$$
\left\{k \frac{\partial}{\partial z}+\frac{y^{2}}{z} \frac{\partial^{2}}{\partial y^{2}}\right\} G_{2}(z, y)=0
$$

Using the Ward identities

$$
\begin{array}{r}
\sum_{n=1}^{N}\left(y_{n}^{l+1} \frac{\partial}{\partial y_{n}}-(l+1) j_{n} y_{n}^{l}\right)<\Phi^{j_{1}}(1) \ldots \Phi^{j_{n}}(n)>=0 \\
l=-1,0,+1 \quad(1)=\left(z_{1}, y_{1}\right), \ldots,(n)=\left(z_{n}, y_{n}\right)
\end{array}
$$

one gets for $j_{n}=0$ very simple constraints

$$
\sum_{n=1}^{N} y_{n}^{l+1} \frac{\partial}{\partial y_{n}} G(1 \ldots N)=0, \quad l=-1,0,+1
$$

and from $l=0$ constraint we see that $y \frac{\partial}{\partial y} G_{2}(z, y)=$ 0 which means that $\frac{\partial}{\partial z} G_{2}(z, y)=0$ and the two-point function is a constant.

For $N=4$ the Ward identities (12) and similar Virasoro $L_{ \pm 1}, L_{0}$ constraints preserve the invariance of correlation functions under projective transformations in $z$ and $y$ planes. Hence the four point function $G(1, \ldots 4)$ depends only on the anharmonic ratios $z$ and $t$ where

$$
z=\frac{z_{32} z_{41}}{z_{31} z_{42}}, \quad t=\frac{y_{32} y_{41}}{y_{31} y_{42}}
$$

and Eq. (9) for $G(1, \ldots 4)=\mathcal{F}(z, t)$ is simplified by the projective nvariance

$$
-\frac{t^{2}}{z} \partial_{t}\left[(t-1) \partial_{t} \mathcal{F}\right]+\frac{(t-1)^{2}}{z-1} \partial_{t}\left(t \partial_{t} \mathcal{F}\right)+k \partial_{z} \mathcal{F}=0
$$

Let us note that this equation is invariant under transformation $z \rightarrow 1-z, t \rightarrow 1-t$, which means that for any solution $\mathcal{F}(z, t)$ there is another one $\mathcal{F}(1-z, 1-t)$.

One particularly simple solution for the holomorphic part is given by

$$
G(1, \ldots 4)=A \ln \left(z / t^{k}\right)+B \ln \left[(z-1) /(t-1)^{k}\right]
$$

We see that we have logarithmic singularities in this correlation function. Moreover, besides logarithms of $z$ we also have logarithms of $t$. The former ones are due to the Jordan cell structure of the Virasoro algebra [3] whereas the latter ones are due to the Jordan cell structure of the Kac-Moody algebra [6], [7]. Hence expansion (8) must be modified

$$
\Phi^{(j)}(y, z)=\sum_{k} y^{j+k}\left[\Phi_{k}^{(j)}(z)+\tilde{\Phi}_{k}^{(j)}(z) \ln y\right]
$$

One can choose constants $A$ and $B$ to get two types of invariant solutions: for $B=0$ we get the solution which changes sign under permutation $z, t \rightarrow 1 / z .1 / t$ which corresponds to the permutation $1 \rightarrow 2$ or $3 \rightarrow 4$ :

$$
\left\langle F_{1}(1) F_{1}(2) F_{2}(3) F_{2}(4)\right\rangle=\ln \left|z / t^{k}\right|
$$

Choosing $A=-2 B$ we get the solution with is invariant under $z, t \rightarrow z^{-1}, t^{-1}$ :

$$
\begin{array}{r}
\left\langle B_{1}(1) B_{1}(2) B_{2}(3) B_{2}(4)\right\rangle= \\
\ln \left[\frac{|z|}{|1-z|^{2}}\right]-\ln \left[\frac{|t|^{k}}{|1-t|^{2 k}}\right]
\end{array}
$$

It is easy to check that there are no solutions invariant under permutations of all operators. Therefore we are lead to the conclusion that there are two types of fermionic fields $F_{a}$ and two types of bosonic fields $B_{a}(a=1,2)$ such that their 4-point correlation functions are given by Eqs.(16, 17). Hence there is a hidden $S U(2)$ symmetry in this problem.

From (16) we derive the following Operator Product Expansion (OPE) for $F_{1}, F_{2}$ :

$$
\begin{array}{r}
F_{a}\left(z+\epsilon_{z}, y+\epsilon_{y}\right) F_{b}(z, y)=\epsilon_{a b}<F F> \\
+\epsilon_{a b}\left[\ln \left|\epsilon_{z}\right| C_{z}(z, y)+\ln \left|\epsilon_{y}\right| C_{y}(z, y)+D(z, y)+\ldots\right]
\end{array}
$$

where the $\epsilon_{a b}$ structures are dictated by $S U(2)$ symmetry and dots stand for terms vanishing at $\epsilon \rightarrow 0$. From this $\mathrm{OPE}$ one can see that the fusion of $F_{1}$ and $F_{2}$ generates a logarithmic triad $C_{z}, C_{y}, D$ :

$$
\begin{array}{r}
\left\langle C_{a} C_{b}\right\rangle=0, \\
\left\langle C_{z}(1) D(2)\right\rangle=1, \quad\left\langle C_{y}(1) D(2)\right\rangle=-k, \\
\langle D(y, z) D(0,0)\rangle=2 \ln \left|y^{k} / z\right|
\end{array}
$$

Let us note that this triad actually represents two pairs: the Virasoro logarithmic pair [3] $C_{z}, D$ which is mixed under the conformal transformation $z \rightarrow \lambda z$ as 


$$
D \rightarrow D-\ln \lambda C_{z}, \quad C_{z} \rightarrow C_{z}
$$

and the Kac-Moody logarithmic pair [6] [7] $C_{y}, D$ which is mixed under the $\mathrm{SL}(2, \mathrm{R})$ transformation $y \rightarrow \epsilon y$ generated by $t^{3}=y(\partial / \partial y)$ :

$$
D \rightarrow D-\ln \epsilon C_{y}, \quad C_{y} \rightarrow C_{y}
$$

For the bosonic operators one gets the following OPE from 4-point function (17):

$$
\begin{aligned}
& B_{a}\left(z+\epsilon_{z}, y+\epsilon_{y}\right) B_{b}(z, y)=\left(2 \delta_{a b}-1\right) \\
& \times\left[\ln \left|\epsilon_{z}\right| C_{z}(z, y)+\ln \left|\epsilon_{y}\right| C_{y}(z, y)+D(z, y)+\ldots\right]
\end{aligned}
$$

In this paper we would like to concentrate on the fermionic sector only. The role of bosonic fields $B_{a}$ and they relation to the operators $\Psi_{0}$ used in [1] will be discussed elsewhere.

It is logical to suggest that $\left\langle C_{a}\right\rangle=0$. Therefore the two-point correlation function of $F_{a}$ is constant which is consistent with the result derived from Eq.(10). To get a non-trivial coordinate dependence one has to define the new two-point function putting in it a vacuum insertion of $D$ :

$$
\left\langle F_{1}(1) F_{2}(2) D(\infty)\right\rangle=\ln \left|z_{12} / y_{12}^{k}\right|
$$

We also can study the 6-point functions, in which case the answer can be presented as an antisymmetrized product of 2-point functions and 4-point functions :

$$
\begin{aligned}
& \left\langle F_{1}(1) F_{1}(2) F_{1}(3) F_{2}(4) F_{2}(5) F_{2}(6)\right\rangle= \\
& +\left\langle F_{1}(1) F_{2}(6)\right\rangle\left\langle F_{1}(2) F_{1}(3) F_{2}(4) F_{2}(5)\right\rangle \\
& -\left\langle F_{1}(1) F_{2}(5)\right\rangle\left\langle F_{1}(2) F_{1}(3) F_{2}(4) F_{2}(6)\right\rangle \\
& -\left\langle F_{1}(1) F_{2}(4)\right\rangle\left\langle F_{1}(2) F_{1}(3) F_{2}(6) F_{2}(5)\right\rangle \\
& -\left\langle F_{1}(2) F_{2}(6)\right\rangle\left\langle F_{1}(1) F_{1}(3) F_{2}(4) F_{2}(5)\right\rangle \\
& +\left\langle F_{1}(2) F_{2}(5)\right\rangle\left\langle F_{1}(1) F_{1}(3) F_{2}(4) F_{2}(6)\right\rangle \\
& +\left\langle F_{1}(2) F_{2}(4)\right\rangle\left\langle F_{1}(1) F_{1}(3) F_{2}(6) F_{2}(5)\right\rangle \\
& -\left\langle F_{1}(3) F_{2}(6)\right\rangle\left\langle F_{1}(2) F_{1}(1) F_{2}(4) F_{2}(5)\right\rangle \\
& +\left\langle F_{1}(3) F_{2}(5)\right\rangle\left\langle F_{1}(2) F_{1}(1) F_{2}(4) F_{2}(6)\right\rangle \\
& +\left\langle F_{1}(3) F_{2}(4)\right\rangle\left\langle F_{1}(2) F_{1}(1) F_{2}(6) F_{2}(5)\right\rangle
\end{aligned}
$$

Using the fact that the two-point functions $\left\langle F_{1}(i) F_{2}(j)\right\rangle$ is a constant (it may be even zero, but we do not know this with certainty and this is not important) and the four-point function is given by (16)

$$
\left\langle F_{1}(a) F_{1}(b) F_{2}(c) F_{2}(d)\right\rangle=\ln \left|\frac{z_{b c} z_{a d}}{z_{a c} z_{b d}}\right|-k \ln \left|\frac{y_{b c} y_{a d}}{y_{a c} y_{b d}}\right|
$$

we can easily see that the six-point function is identically zero. The proof of this is the following. We can see that each pair of indices $a$ and $b$ where the first one is $1,2,3$ and the second one is 4,5,6 appears precisely in four terms in (24). For example the pair 3, 4 appears in a first, second, fourth and fifth four-point correlation functions, which means that the terms $\ln \left|z_{34}\right|$ and $\ln \left|y_{34}\right|$ will enter four times. A simple inspection of (24) shows that two times they enter with sign plus and two times with sign minus and the total contribution is zero. In the same way we can check each pair of indices, for example the pair 1,5 enters fourth, six, seventh and ninth correlation functions and again the total contribution is zero, etc.

Thus we see that the the six-point correlation function is zero. It is not difficult to generalize these solutions for general $N=4 M$ and $N=4 M+2$. In the first case one has to decompose $N$ points in groups of four in all possible ways and take a symmetrized (bosons) or antisymmetrized (fermions) sum of products of solutions (14). In the second case we multiply this by two-point correlation functions $\left\langle F_{1} F_{2}\right\rangle$, but since they are constants it is essentially the same procedure. One can show that for fermions all these correlation functions are identically zero. We have checked it explicitly for 8-point functions. The structure of antisymmetrization in a higher dimensional case is such that each pair appears in an even number of four-dimensional correlation functions half of which have negative signs.

It is most remarkable that all higher-order fermionic correlation functions vanish. Since the $C, D$-operators of the triad can be generated via fusions of the fermions (18), this result means that all higher-order correlation functions of the logarithmic fields vanish. Not only threepoint functions are trivial

$$
\begin{array}{r}
\left\langle C_{a} C_{b} C_{c}\right\rangle=0, \quad\left\langle C_{a} C_{b} D\right\rangle=0, \\
\left\langle C_{a} D D\right\rangle=0,\langle D D D\rangle=0
\end{array}
$$

where indices $a, b, c$, for zero norm operators $C$ means $y$ or $z$, but also all four-point functions are

$$
\begin{aligned}
\langle C C C C\rangle=0, & \langle C C C D\rangle=0, & \langle C C D D\rangle & =0, \\
& \langle C D D D\rangle=0, & & \langle D D D D\rangle=0,
\end{aligned}
$$

From here we can dedudce that OPE of $C$ and $D$ may have only the $C$ operator (besides unity operator $I$ ):

$$
\begin{aligned}
C_{a} C_{b} & =F_{a b c} C_{c} \\
C_{a} D & =<C_{a} D>I+F_{a b} C_{b} \\
D D & =<D D>I+F_{a} C_{a}
\end{aligned}
$$

This is precisely what one needs to be able to truncate an expansion in superpositions of $C$ and $D$ operators. Adding a new $C$ or $D$ term inside a correlation function does not change its scaling behaviour; only the first two $C$ and $D$ operators matter, not the next ones. Thus we conclude that model (12) remains conformally invariant when one adds to the action the term

$$
\eta \int d^{2} x: F_{1} F_{2}:
$$

It is quite a challenge to fully understand the physical meaning of the operators discussed in this paper. 
The reason is that our knowledge of relationship between observables in the Quantum Hall problem and fields of model (11) is very limited. The only thing we can be sure about is that such operators are important. This certainty is supported by results obtained for other disordered systems (see [9], [10]), where operators with zero scaling dimensions also play very important role. Hopefully, better understanding of mathematical structure of these theories will help to get an insight into their physical meaning. It is also possible that these operators will play an important role in other physical problems where $S L(2 . R)$ WZNW model is important, for example, for gravitational dressing of exactly integrable models and logarithmic operators in string theory [5].

[1] M. J. Bhaseen, I. I. Kogan, O. A. Soloviev, N. Taniguchi and A. M. Tsvelik, cond-mat-9912060.

[2] J.-S. Caux, N. Taniguchi, and A. M. Tsvelik, Phys. Rev. Lett. 80, 1276 (1998); Nucl. Phys. B525, 621 (1998). J.-S. Caux, Phys. Rev. Lett. 81, 4196 (1998). B

[3] V. Gurarie, Nucl. Phys. B410, 535 (1993).

[4] J.-S. Caux, I. I. Kogan and A. M. Tsvelik, Nucl. Phys. B466, 444 (1996).

[5] A. Bilal and I. I. Kogan, hep-th/9407151, Princeton University preprint PUPT-1482 (unpublished); Nucl.Phys. B 449, 569 (1995); I. I. Kogan and N.Mavromatos, Phys. Lett. B375 (1996), 111

[6] I. I. Kogan, A. Lewis, and O. A. Soloviev, Int. J. Mod. Phys. A13, 1345 (1998)

[7] I. I. Kogan and A. Lewis, Nucl. Phys. B509, 687 (1998).

[8] V. Fateev and A. Zamolodchikov, Sov. Nucl. Phys. 43, 1031 (1986).

[9] V. Gurarie and A. W. W. Ludwig, cond-mat/9911392.

[10] J. L. Cardy, cond-mat/9911457. 